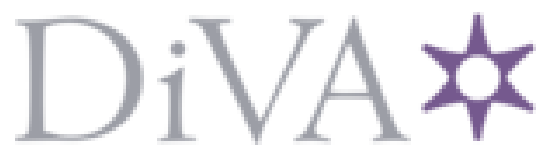

http://www.diva-portal.org

Postprint

This is the accepted version of a paper presented at 2019 IEEE Intelligent Transportation Systems.

Citation for the original published paper:

Liu, T., Feng, L., Hellgren, M., Wikander, J. (2019)

A Binary Controller to Ensure Engine Peak Efficiency for aParallel Hybrid Electric Car In: 2019 IEEE Intelligent Transportation Systems (pp. 726-732). Auckland, New Zealand

N.B. When citing this work, cite the original published paper.

Permanent link to this version:

http://urn.kb.se/resolve?urn=urn:nbn:se:kth:diva-268543 


\title{
A Binary Controller to Ensure Engine Peak Efficiency for a Parallel Hybrid Electric Car*
}

\author{
Tong Liu, Lei Feng, Mikael Hellgren, and Jan Wikander
}

\begin{abstract}
The fuel efficiency of the hybrid electric vehicle (HEV) highly relies on the engine efficiency. This paper proposes a particular powertrain configuration on a small hybrid electric racing car where the engine is only allowed to operate with peak efficiency. An online binary controller, accordingly, is developed based on dynamic programming (DP) to control the engine on/off status and the torques from the electric motors (EMs) to ensure the HEV can successfully complete the drive mission with minimal fuel consumption. For comparison, the paper also develops an optimal powertrain controller of the same HEV with the normal usage of the engine, i.e., the engine operates at any feasible point of the 2D fuel efficiency map. The simulation results show that this binary controller can improve roughly $13 \%$ on fuel efficiency compared with the general engine case.
\end{abstract}

Keywords - hybrid electric vehicle, energy management control, engine peak efficiency

\section{INTRODUCTION}

A common limitation of the state-of-the-art hybrid electric vehicle (HEV) design and control methods is to simply reuse the conventional combustion engine with smaller horsepower [1], [2]. The engine's characteristics are not altered for its new working environment. When used as the only power source, the engine must have balanced fuel consumption and tailpipe emission in all working condition. This tradeoff compromises the peak value of the high efficiency which occurs in a narrow range of the engine torque and speed. By contrast, when coupled with electric motors (EMs) in the hybrid powertrain, the engine will mostly operate in a small range with high efficiency and the EMs can provide the complementary driving power [3], [4]. Consequently, one can redesign the engine to maximize the fuel efficiency meanwhile minimize the exhaust emission for the small working range without worrying about worse performance outside this range.

A prerequisite of the redesigned engine is that the engine must be prevented from operating outside the designed operation range. This requires adequate torque and power from the EM and the electrical energy storage (EES) whenever the engine cannot meet the torque demand [5], [6]. The guarantee of the available electrical power in the new hybrid powertrain is much stronger than that in the conventional HEV. This requires improvements on topology, sizing and control of the $\mathrm{EM}$, the EES and the transmission to ensure that the HEV can always satisfy the torque and power demand from the driver [7], [8]. This paper focuses on the control aspect to evaluate the feasibility of the new type of hybrid electric powertrain.

For a specially developed small hybrid electric car, we evaluate if we can develop an optimal energy management

*The project is financially supported by Chinese Scholarship Council (CSC), and Swedish Electromobility Centre (SEC) in the thematic area of system studies and methods. The project title is "Optimal Integration of Combustion Engines and Electric Motors for HEVs". controller that limits the engine to work only in the narrow region with peak efficiency. If the controller can be developed to reach the similar driving performance and comparable fuel efficiency, the result could indicate that the specially designed engine is feasible to replace the general-purpose engine.

The selected HEV is a prototype racing car with a parallel powertrain including three propulsion components: a small gasoline engine, a BLDC motor, and a small DC motor, shown in Fig. 1. The BLDC motor can work as either an actuator or a generator, and the DC motor can only provide limited amount of positive power. After a special refit, the DC motor works more efficiently than the BLDC motor and is connected to the drive shaft without clutch. To ensure peak efficiency, the engine's throttle is controlled based on its rotational speed so that it always reaches the peak efficiency. In addition, a supercapacitor, rather than a battery, is selected as the EES mainly because the higher specific power is more important than the higher energy density for this racing HEV.

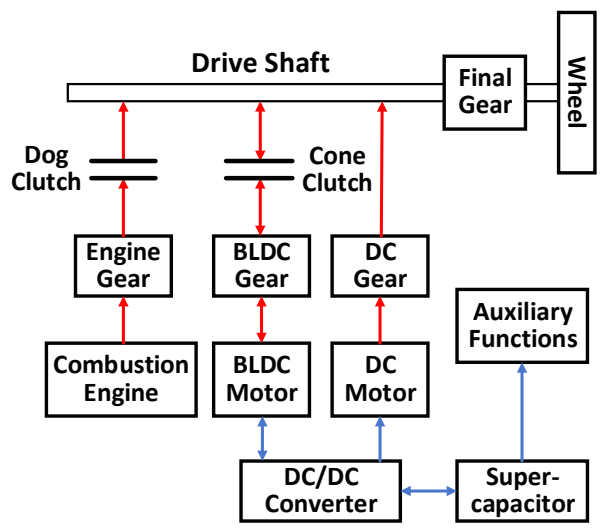

Figure 1. Powertrain Architecture of the Hybrid Electric Car

In Shell Eco-marathon [9], [10], each competing vehicle must finish a prescribed uneven circular track several loops within the allowable time limit. The vehicle must stop at the end of every loop. After the last loop, if the EES state of charge (SOC) is less than its initial value, the engine must be turned on to recharge the EES until the SOC is equal to its initial value. After that, the total fuel consumption is the index to estimate the HEV's performance.

The HEV racing problem is formulized as an optimal energy management control problem of a dynamic process [11], [12]. Plenty of control approaches have been developed to solve similar problems. Among those, fuzzy logic [7], [13], and deterministic rule-based methods [14], [15], are widely used in online control due to their low cost on development,

T. Liu, L. Feng, M. Hellgren, and J. Wikander are with KTH Royal Institute of Technology, Department of Machine Design, Brinellvägen 83, Stockholm, 10044, Sweden. L. Feng is the corresponding author. e-mail: \{tongliu, lfeng, hellgren, janwi\}@kth.se 
implementation, and computation overhead; but they cannot ensure optimal performance or robustness. Model predictive control (MPC) [16], [17], Pontryagin's minimum strategy (PMP) [18], [19], and equivalent consumption minimization strategy (ECMS) [20], [21], are typical methods of real-time optimal control and can obtain a performance close to the optimum; however, MPC highly relies on the prediction accuracy while PMP and ECMS require a large effort of "tuning and calibration" to refine co-state or equivalence factor. As an emerging method recently, reinforcement learning [22], [23], does not require accurate models of the dynamic process and the environment. In spite of this, the reinforcement process takes lots of time and needs "big data" for complex systems with multiple degrees of freedom (DOF). The true optimal solution of the HEV control can be obtained by global optimization methods, such as dynamic programming (DP) [24], [25] and simulated annealing algorithm [26]; but they rely on a prior knowledge of the entire driving mission and lead to tremendous computation overhead. These methods, therefore, are often used as benchmarks to evaluate the performances of other online control methods.

To investigate the feasibility and the advantage on fuel efficiency of the limited usage of the engine in HEVs, this paper develops two optimal controllers based on DP to calculate the best fuel efficiency of the HEV using both the limited engine and the general engine respectively. By this way, the car with the limited engine can achieve a fuel efficiency $13 \%$ higher than the one of general engine.

The reminder of this paper is organized as follows. Section II introduces the HEV's dynamics and formulates its one loop racing into an optimal control problem; The methodology of DP based controller design is described in Section III; simulation results and detailed analysis are carried out in Section IV; Section V concludes the paper and suggests the future work.

\section{PROBLEM FORMULATION}

This section formalizes the energy management control problem of this hybrid electric racing car as a nonlinear optimal control problem with constraints. Based on this modelled dynamic process, a sequence of control decisions regarding the engine on/off status as well as the torques of the engine and EMs will be calculated to drive the HEV finishing the driving task with minimum fuel cost.

\section{A. Vehicle Longitudinal Dynamics}

Denote $T_{\text {tra }}$ as the traction torque at the drive wheel. The longitudinal dynamic equation is

$$
\begin{aligned}
\delta m_{\text {gross }} & \dot{v}_{\text {veh }}(t)= \\
& \frac{1}{r_{\text {veh }}} T_{\text {tra }}(t)-F_{\text {grade }}(t)-F_{\text {roll }}(t)-F_{\text {aero }}(t)
\end{aligned}
$$

where $m_{\text {gross }}$ is the gross mass including the entire vehicle and the driver; $\delta$ is the equivalent mass ratio $(>1)$ reflecting the effect on acceleration from the inertial of the powertrain; $r_{v e h}$ is the radius of the vehicle tire; whilst the vehicle's longitudinal velocity $v_{v e h}$ is the derivative of the vehicle's displacement $y_{v e h}$.

The aerodynamic resistance $F_{\text {aero }}$ is solely determined by $v_{v e h}$, and $y_{v e h}$ affects the road gradient force $F_{\text {grade }}$ and $F_{\text {roll }}$ via its associative road slope angle $\alpha\left(y_{v e h}\right)$. The relevant formulae (2)-(4) are given with several parameters referring to a specific vehicle (namely the windward area $A_{f}$, the air drag coefficient $C_{d}$, and the rolling resistance coefficient $C_{r}$ ) along with the gravity acceleration $g$ and the air density $\rho_{\text {air }}$.

$$
\begin{gathered}
F_{\text {aero }}(t)=\frac{1}{2} \rho_{\text {air }} A_{f} C_{d} v_{\text {veh }}^{2}(t) \\
F_{\text {grade }}(t)=m_{\text {gross }} g \sin \alpha\left(y_{\text {veh }}(t)\right) \\
F_{\text {roll }}(t)=C_{r} m_{\text {gross }} g \cos \alpha\left(y_{\text {veh }}(t)\right)
\end{gathered}
$$

To a parallel HEV, the traction torque is the combination of the torque from the fuel and electric paths, $T_{\text {fuel }}$ and $T_{\text {elec }}$, as follows.

$$
\begin{aligned}
& T_{\text {tra }}(t)= \\
& \begin{cases}\left(T_{\text {fuel }}(t)+T_{\text {elec }}(t)\right) \eta_{\text {tra }} R_{p} ; & T_{\text {elec }}(t)>0 \\
\left(T_{\text {fuel }}(t) \cdot \eta_{\text {tra }}+T_{\text {elec }}(t) / \eta_{\text {tra }}\right) R_{p} ; & T_{\text {elec }}(t)<0\end{cases}
\end{aligned}
$$

where $R_{p}$ is the final gear ratio; and $\eta_{\text {tra }}$ is the transmission efficiency in average.

\section{B. Powertrain Dynamics}

When the engine is turned on and the clutch fully engaged, $T_{f u e l}$ is the product of the torque output from the engine $T_{\text {eng }}$ and the gear ratio between the engine and the driveline $R_{\text {eng }}$.

$$
T_{\text {fuel }}(t)=T_{\text {eng }}(t) R_{\text {eng }}
$$

The transient engine net efficiency $\eta_{\text {eng }}$ is defined as

$$
\begin{gathered}
\eta_{\text {eng }}(t)=\frac{P_{\text {eng }}(t)}{P_{\text {fuel }}(t)}=\frac{\omega_{\text {eng }}(t) T_{\text {eng }}(t)}{\dot{m}_{\text {fuel }}(t) Q_{\text {fuel }}} \\
\omega_{\text {eng }}(t)=\frac{v_{\text {veh }}(t)}{r_{\text {veh }}} R_{p} R_{\text {eng }}
\end{gathered}
$$

where $P_{\text {eng }}(t)$ are $P_{\text {fuel }}(t)$ are the engine output power and fuel power respectively, $\omega_{\text {eng }}(t)$ is the angular velocity of the crankshaft, $\dot{m}_{f u e l}$ is the mass consumption ratio of a specific fuel whose low heat value is $Q_{f u e l}$.

The total fuel consumption $M_{\text {fuel }}$ to be optimized during the driving process can be calculated by

$$
M_{\text {fuel }}=\int_{0}^{T} \dot{m}_{\text {fuel }}(\tau) d \tau \text {. }
$$

Similarly, to match the wheel's velocity with the motor's spinning speed, both DC and BLDC motors are connected to the drive shaft through distinctive transmission ratios $R_{D C}$ and $R_{B L D C}$. Therefore, $T_{\text {elec }}$ is written as

$$
T_{\text {elec }}(t)=T_{D C}(t) R_{D C}+T_{B L D C}(t) R_{B L D C}
$$

where $T_{D C}$ is the torque output from the DC motor which is always a non-negative value, while the torque output from the BLDC motor $T_{B L D C}$ can be either positive or negative.

\section{Electrical System Dynamics}

The currents to drive the DC motor and the BLDC motor are defined as $I_{D C}$ and $I_{B L D C}$ respectively. The energy efficiencies of the two EMs are $\eta_{D C}\left(\omega_{D C}(t), T_{D C}(t)\right)$ and $\eta_{B L D C}\left(\omega_{B L D C}(t), T_{B L D C}(t)\right)$, both of which are determined by the combination of speed and torque. For simplicity, the two efficiency functions are denoted as $\eta_{D C}(t)$ and $\eta_{B L D C}(t)$. The 
dynamics of the EES, a supercapacitor in this project, is described by (11)-(17).

$$
I_{D C}(t)=\frac{\omega_{D C}(t) T_{D C}(t)}{V_{c a p}(t) \eta_{D C}(t)}
$$

$I_{B L D C}(t)=$

$$
\begin{aligned}
& \begin{cases}\frac{\omega_{B L D C}(t) T_{B L D C}(t)}{V_{c a p}(t) \eta_{B L D C}(t)} ; & T_{B L D C}(t)>0 \\
\frac{\omega_{B L D C}(t) T_{B L D C}(t) \eta_{B L D C}(t)}{V_{c a p}(t)} ; & T_{B L D C}(t)<0\end{cases} \\
& \omega_{D C}=\frac{v_{v e h}(t)}{r_{v e h}} R_{p} R_{D C} \\
& \omega_{B L D C}=\frac{v_{v e h}(t)}{r_{v e h}} R_{p} R_{B L D C} \\
& I_{\text {cap }}(t)=I_{B L D C}(t)+I_{D C}(t)+P_{a u x} / V_{c a p}(t) \\
& \dot{V}_{\text {cap }}(t)= \begin{cases}-\frac{I_{\text {cap }}(t)}{C \eta_{\text {cap }_{\text {dis }}}(t)} ; & I_{\text {cap }}(t)>0 \\
-\frac{I_{\text {cap }}(t) \eta_{\text {cap }}(t)}{C} ; & I_{\text {cap }}(t)<0\end{cases} \\
& \operatorname{SOC}(t)=\frac{C V_{\text {cap }}(t)}{Q_{0}}
\end{aligned}
$$

where $V_{c a p}$ is the bus voltage of the supercapacitor; $I_{\text {cap }}$ is the current flowing across the supercapacitor; $P_{\text {aux }}$ refers to the average electrical power to the on-board auxiliary devices; $C$ is the nominal capacitance; and $\eta_{c a p_{d i s}}$ as well as $\eta_{c a p_{\text {cha }}}$ refer to the Coulombic efficiency coupled with $V_{c a p}$ in discharging and charging modes. Note that $S O C$ is a general index to estimate the EES electricity storage. For easy measurement, $V_{c a p}$ is selected as an indicator to $S O C$, while $Q_{0}$ is the nominal charge capacity.

\section{Optimal Control Problem Formulation for the HEV with General Engine}

This section formulates the cost function and the vehicle dynamics in discrete-time format. The competition rule is to finish the trip within a time limit by consuming minimal amount of fuel. The speed trajectory along the trip is not prescribed, but instead will be found out as the consequence of the optimal control. Since the actual time to finish the driving task is unknown a priori, we discretize the trip by even distance $\Delta y$ instead of even time as the step interval. The accumulated driving time $t$ is treated as a state variable. Thus, the HEV model is converted into a dynamic model with 3 state variables and 3 control variables in (18)-(20).

$$
\mathbf{x}(k+1)=f(\mathbf{x}(k), \mathbf{u}(k))
$$

subject to

$$
\begin{gathered}
\mathbf{x}=\left[\begin{array}{lll}
v_{\text {veh }} & t & V_{\text {cap }}
\end{array}\right]^{T} \\
\mathbf{u}=\left[\begin{array}{lll}
T_{\text {eng }} & T_{D C} & T_{B L D C}
\end{array}\right]^{T}
\end{gathered}
$$

To be explicit, (18) can be expanded by (21)-(25).

$$
\begin{gathered}
v_{v e h}(k+1)=\sqrt{v_{v e h}(k)^{2}+2 a_{v e h}(k) \Delta y} \\
t(k+1)=t(k)+\Delta t(k) \\
V_{c a p}(k+1)=V_{c a p}(k)+\Delta V_{\text {cap }}(k)
\end{gathered}
$$

$$
\begin{gathered}
\Delta t(k)=\frac{2 \Delta y}{v_{v e h}(k)+\sqrt{v_{v e h}(k)^{2}+2 a_{v e h}(k) \Delta y}} \\
\Delta V_{c a p}(k)=\dot{V}_{c a p}(k) \Delta t(k)
\end{gathered}
$$

where $a_{v e h}(k)$ is the vehicle acceleration at $k$ th step and determined by (1)-(10); $\Delta V_{c a p}(k)$ is updated according to (11)-(16).

The cost function for consuming minimum fuel and maintaining its electric sustainability is derived as follows:

For all $k$ in $\{0,1,2, \ldots, N-1\}$

$$
\begin{gathered}
\min _{\mathbf{u}(k)} \sum_{k=0}^{N-1} m_{\text {fuel }}(\mathbf{x}(k), \mathbf{u}(k))+\mathcal{W} \Delta E_{\text {cap }}(\mathbf{x}(N)) \\
\Delta E_{\text {cap }}(\mathbf{x}(N))=\frac{C}{2}\left(V_{\text {cap }}(0)^{2}-V_{\text {cap }}(N)^{2}\right)
\end{gathered}
$$

subject to

$$
\begin{aligned}
& 0 \leq v_{\text {veh }}(k) \leq v_{\text {veh }_{\text {max }}} \\
& 0 \leq t(k) \leq t_{\max }
\end{aligned}
$$

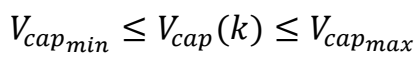

$$
\begin{aligned}
& T_{\text {eng }}\left(v_{\text {min }}(k)\right) \leq T_{\text {eng }}(k) \leq T_{\text {eng }}\left(v_{\text {max }}\left(v_{\text {veh }}(k)\right)\right. \\
& 0 \leq T_{D C}(k) \leq T_{D C_{\max }}\left(v_{v e h}(k)\right) \\
& T_{B L D C_{\text {min }}}\left(v_{v e h}(k)\right) \leq T_{B L D C}(k) \leq T_{B L D C_{\text {max }}}\left(v_{\text {veh }}(k)\right) \\
& \mathbf{x}(0)=\left[\begin{array}{lll}
0 & 0 & V_{\text {cap }_{\text {init }}}
\end{array}\right]^{T}
\end{aligned}
$$

where $\Delta E_{c a p}(\mathbf{x}(N))$ implies the electric charge deviation during the driving process and works as a penalty to the electric sustain; $\mathcal{W}$ is a constant concerning the specific fuelelectricity conversion ratio in average; $(\cdot)_{\max }$ and $(\cdot)_{\min }$ are the defined boundary values for each correlative variable (to state variables, they are constant; yet to control variables, they may be variables coupled with $\left.v_{v e h}\right) ; V_{\text {cap }}$ init is a tunable parameter as the initial state of $V_{\text {cap }}$, as long as it locates between its upper and lower boundaries.

\section{E. Optimal Control Problem Formulation for the HEV with Binary Engine Control to Ensure Peak Efficiency}

This section presents an optimal control problem referring to the HEV with the redesigned engine which is controlled to be only on/off. When the engine is on, it must operate at its peak efficiency point corresponding to the current engine speed. These peak efficiency points determine a curve in the 2D efficiency map, as illustrated in Fig. 8 by the blue curve.

The formulae about the electric path are identical to those of general engine defined above; the only difference on the fuel path is shown as:

$$
T_{\text {fuel }}(t)= \begin{cases}T_{\text {eng }}^{*}\left(\omega_{\text {eng }}(t)\right) R_{\text {eng }} ; & S_{\text {eng }}=1 \\ 0 ; & S_{\text {eng }}=0\end{cases}
$$

where $T_{e n g}^{*}$ is not a flexible variable but determined by $\omega_{\text {eng }}$; $S_{\text {eng }}$ is a binary variable representing the current state of the engine ("1" means on and " 0 " off).

Even though the engine does not consume any fuel after turned off, the action of switching on/off the engine itself will consume extra energy which cannot be neglected. In addition, when the engine is switched on, there is also a time delay before the torque is transmitted to the wheel. Moreover, 
frequent switching on/off is unacceptable to either vehicle drivability or engine lifespan. Thus, the engine state is treated as both a control variable $S_{\text {eng_con }}$ and an extra state variable $S_{\text {eng. }}$. In discrete-time domain, we define it like that:

$$
S_{\text {eng }}(k+1)=S_{\text {eng_con }}(k)
$$

Furthermore, $T_{\text {fuel }}$ is refined and an extra equivalent fuel cost on engine switching is given as:

$$
\begin{gathered}
T_{\text {fuel }}(k)=S_{\text {eng }}(k) T_{\text {eng }}^{*}\left(\omega_{\text {eng }}(k)\right) R_{\text {eng }} \\
m_{\text {sw }}(k)= \begin{cases}0 ; & S_{\text {eng_con }}(k)=S_{\text {eng }}(k) \\
m_{\text {eng }} ; & S_{\text {eng_con }}(k) \neq S_{\text {eng }}(k)\end{cases}
\end{gathered}
$$

where $m_{e n g}$ is the mass of the equivalent fuel consumption reflecting the real fuel and the extra electricity cost to switch engine on/off once; $S_{\text {eng }}$ and $S_{\text {eng_con }}$ determine if the real $\operatorname{cost} m_{s w}$ existing or not. Consequently, a new HEV model of 4 state variables and 3 control variables are established.

$$
\begin{gathered}
\mathbf{x}=\left[\begin{array}{llll}
v_{\text {veh }} & t & V_{\text {cap }} & S_{\text {eng }}
\end{array}\right]^{T} \\
\mathbf{u}=\left[\begin{array}{lll}
S_{\text {eng_con }} & T_{D C} & T_{B L D C}
\end{array}\right]^{T}
\end{gathered}
$$

The optimal control problem can be updated as follow:

$$
\begin{array}{r}
\min _{\mathbf{u}(k)} \sum_{k=0}^{N-1} m_{\text {fuel }}(\mathbf{x}(k), \mathbf{u}(k))+m_{\text {sw }}(\mathbf{x}(k), \mathbf{u}(k))+ \\
\mathcal{W} \Delta E_{\text {cap }}(\mathbf{x}(N))
\end{array}
$$

Equations (27)-(34) except (31) are still valid for this case. Some extra constraints are declaimed as supplement.

$$
\begin{aligned}
S_{\text {eng_con }}(k) & \in\{0,1\} \\
S_{\text {eng }}(0) & =0
\end{aligned}
$$

\section{Control Design By DynAmic Programming}

As long as the road topographical information is given, DP is capable of generating an optimal solution to the HEV energy management problems defined above. In this project, we apply the DP results for online controller design via look-up tables. Therefore, an online control architecture of 2 layers is built up, shown in Fig. 2.

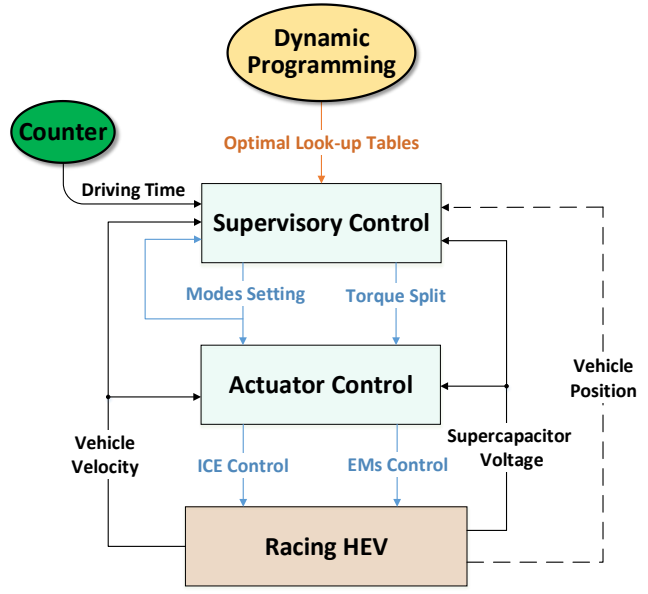

Figure 2. Control Architecture of the Hybrid Electric Car

The whole control design process consists of two parts: offline DP colored by yellow and online control by cyan. Located on top of the online control, DP computes the statebased control strategy that determines the optimal control input at every reachable state determined by (18). This causal relationship is captured by high-dimensional look-up tables. These tables can be used for online control [27], [28]. The online control consists of the supervisory and actuator controls The former one is responsible for allocating each propulsion component an optimized torque assignment and setting them into corresponding operation modes. Located at the bottom layer, the actuator control executes the optimization control decisions on engine and EMs.

\section{A. Solutions by Dynamic Programming}

A generic DP function [29], [30] is employed to solve the global optimal solutions in MATLAB. To apply DP, the continuous state variables and control inputs have to be discretized into finite grid points.

Even conducting an offline computation, the computation time and memory overhead from DP "curse of dimension" is also a huge challenge to a general computer. However, reducing the grid densities, or decreasing the step number $N$ may worsen the performance of the controller. Therefore, pertinent countermeasures are needed to reduce the computation load without obvious performance degradation.

As the driving time $t$ is monotonic, it is wasteful to always use 0 and the maximal time limit as the lower and upper bounds at every step to generate the grid. We use dynamic bounds to significantly reduce the memory usage of DP.

$$
\begin{aligned}
& \text { For all } k \text { in }\{0,1,2, \ldots, N-1\} \\
& \qquad t_{\text {min }}(k)<t(k)<t_{\text {max }}(k) \\
& \qquad t_{\text {max }}(k)=a_{1}+b_{1} \cdot k \\
& t_{\text {min }}(k)= \begin{cases}0 ; & k \leq k_{0} \\
a_{2}+b_{2} \cdot k ; & k>k_{0}\end{cases}
\end{aligned}
$$

where $t_{\min }(k)$ and $t_{\max }(k)$ are the permitted lower and upper boundaries of the driving time at $k$ th step. $a_{1}, a_{2}, b_{1}, b_{2}$ and $k_{0}$ are tunable parameters by conservative forecast according to the HEV's maximal velocity and acceleration along with the left distance of the trip.

Fig. 3 illustrates the optimized results of the driving time for two study cases. Using the dynamical constraint range on the driving time, the searching space is shrunken to a narrow channel (between the purple and green lines). If the discretization boundaries are constant 0 and 310 seconds, we need 311 grid points to reach the step resolution of 1 second. Thanks to the dynamic boundaries, only approximately 20 grid points are needed at each step to reach the same resolution of 1 second.

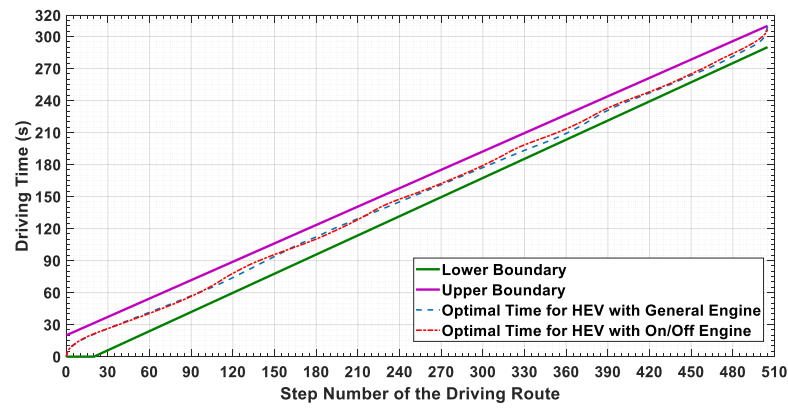

Figure 3. Control Architecture of the Hybrid Electric Car

An additional trick to further decrease computational complexity is to combine two control variables into one. Since 
the two EMs are driven by the same EES, they are virtually combined into one with lumped torque output $T_{E M}$. To realize this, the "new" motor's operation range is assigned by adding the DC motor's range to that of the BLDC motor via the gearbox transmission. Because $T_{D C}$ is non-negative, the new range is not symmetric any more. Since the DC motor is more efficient than the BLDC motor, DC motor is always preferred as an actuator based on which the EM efficiency $\eta_{E M}$ is as well updated on the map. Therefore, the combined motor has the following characteristics.

$$
\begin{gathered}
T_{E M}(k)=T_{B L D C}(k)+T_{D C}(k) \frac{R_{D C}}{R_{B L D C}} \\
T_{B L D C_{\text {min }}}\left(v_{\text {veh }}(k)\right) \leq T_{E M}(k) \\
\leq T_{B L D C_{\text {max }}}\left(v_{\text {veh }}(k)\right)+T_{D C_{\text {max }}}\left(v_{\text {veh }}(k)\right) \cdot \frac{R_{D C}}{R_{B L D C}}
\end{gathered}
$$

Adapting to the definition of the virtual motor, $T_{\text {elec }}(k)$ and $I_{\text {cap }}(k)$ should be modified as below.

$$
\begin{aligned}
& T_{\text {elec }}(k)=T_{E M}(k) R_{B L D C} \\
& I_{\text {cap }}(k)=I_{E M}(k)+P_{\text {aux }} / V_{\text {cap }}(k) \\
& I_{E M}(k)= \begin{cases}\frac{\omega_{B L D C}(k) T_{E M}(k)}{V_{c a p}(k) \eta_{E M}(k)} ; & T_{E M}(k)>0 \\
\frac{\omega_{B L D C}(k) T_{E M}(k) \eta_{E M}(k)}{V_{c a p}(k)} ; & T_{E M}(k)<0\end{cases} \\
& \eta_{E M}(k)=
\end{aligned}
$$

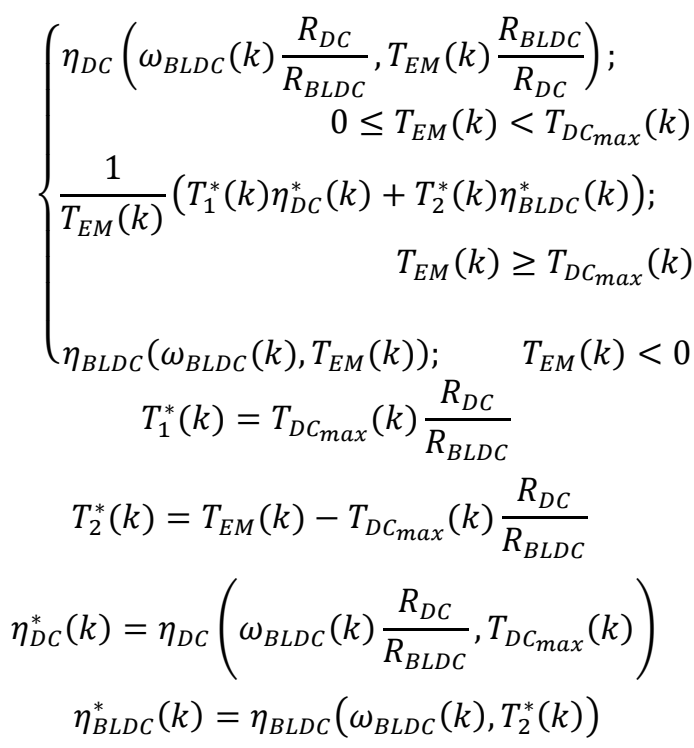

where $T_{1}^{*}, T_{2}^{*}, \eta_{D C}^{*}$, and $\eta_{B L D C}^{*}$ are intermediate variables for calculation.

Although this method causes deviations on estimating SOC under online control, an appropriate online controller should be capable of eliminating such error rapidly. The simulation results confirm this in Section IV. After all, its great contribution to efficient computation is unquestioned.

\section{B. Online Supervisory Control}

To apply the offline control strategy onto real-time energy management, the offline DP results should be collected up sequentially to generate the correlative look-up tables. Each look-up table works as an independent online supervisory controller mapping to a control variable. All the look-up tables are of the same dimensions which equals to number of the state variables plus one.

In this paper, two sets of look-up tables are developed for two different engine configurations. Each set contains two look-up tables.

For the first set to the general engine case, the 4D look-up tables calculate $T_{e n g}$ and $T_{E M}$ by 4 inputs, namely $v_{v e h}, t$, $V_{c a p}$, and $y_{v e h}$. The last element determines, whilst mapping to the offline DP, which step the HEV reaches or between which two steps the HEV is located. Note that only the lumped torque $T_{E M}$ can be calculated from the second look-up table. There should be another algorithm to calculate $T_{D C}$ and $T_{B L D C}$. It only works when $T_{E M}$ is positive because only BLDC motor can provide negative torque. As mentioned above, DC motor is the preferred actuator always. For this reason, this algorithm is not difficult to derive any longer and given below.

$$
\begin{gathered}
T_{D C}(t)=\left\{\begin{array}{lc}
0 ; & T_{E M}(t) \leq 0 \\
T_{E M}(t) \frac{R_{B L D C}}{R_{D C}} ; & 0<T_{E M}(t)<T_{1}^{*}(t) \\
T_{D C_{\max }}(t) ; & T_{E M}(t) \geq T_{1}^{*}(t)
\end{array}\right. \\
T_{B L D C}(t)=\left\{\begin{array}{lc}
0 ; & 0 \leq T_{E M}(t) \leq T_{1}^{*}(t) \\
T_{E M}(t) ; & \text { else }
\end{array}\right.
\end{gathered}
$$

The second set look-up tables, concerning the on/off only engine case, are similar to the first set. However, they are 5D look-up tables that have an extra input $S_{\text {eng }}$. Besides, its first output $S_{\text {eng_con }}$ is a binary value and needs to be rounded to the nearest integer after interpolation.

\section{Actuator Control}

This bottom layer control contains three controllers to execute instantaneous torque split decision on DC motor, BLDC motor, and engine respectively. PID control obtained by pole placement [31], [32], is adopted to develop all these three controllers. For EMs, feedforward control works together with feedback control to provide required voltage by setting the pulse width modulation (PWM). The engine controller determines the engine on/off status and the correct throttle according to the demand from the supervisory controller.

\section{Simulation Result}

To evaluate the performance of the controllers on two engine configurations, simulation testing is conducted on MATLAB/Simulink. A classical circular track from SEM Europe 2016 [33], [34], is illustrated in Fig.4 as the testing route where an HEV is expected to finish 8 loops of 2240 meters in 43 minutes. To compensate the lateral movement, the $\mathrm{HEV}$ is required to finish at least 2262 meters (roughly $1 \%$ margin) in 305 seconds and the deviation of EES terminal voltage to its initial value should be limited within 0.5 volt.

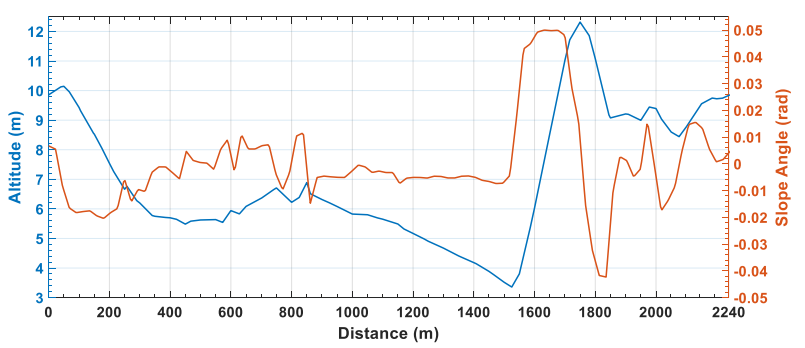

Figure 4. Topographic Information of SEM Europe 2016 
For comparison, some important performance indexes, including the vehicle velocity trajectory, EES voltage variation, and engine on/off status along with working efficiency, are presented. Based on these, the equivalent fuel efficiencies to different control methods, as the unit $\mathrm{km} / \mathrm{L}$ (gasoline), are calculated.

\section{A. Vehicle Velocity Trajectory}

In the plots below, the blue curves depict the results of controlling the engine continuously, and the red curves show the results of the on/off control of the engine. The velocity trajectories under different control methods are shown in Fig. 5. Compared to the general configuration, the on/off only engine will result in a more rugged and fluctuant profile nearly independent of the road slope angle. The optimal control of the on/off engine is similar to the classical bang-bang control. When the engine is on, it accelerates the vehicle to the closeto-maximal velocity. When the engine is off, the vehicle rolls freely or is propelled by EMs. The large oscillation in speed may not be a crucial problem to a racing car, but is problematic for normal passenger cars.

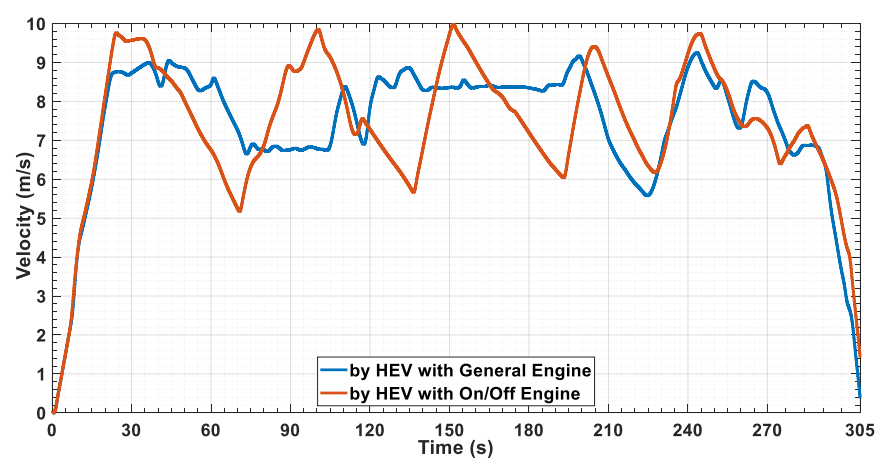

Figure 5. Velocity Trajectories of the HEVs with Two Different Engines

\section{B. EES Voltage Variation}

In this testing case, the initial voltage of the supercapacitor is set as 48 volts and thus the terminal lower boundary is $\mathbf{4 7 . 5}$ volts. Shown in Fig. 6, both results meet this requirement, but more electrical energy is recuperated from the engine and the regenerative brake under the engine on/off control. Note that when the optimal engine torque is larger than the value to drive the vehicle, the excess torque drives the BLDC motor to charge the supercapacitor.

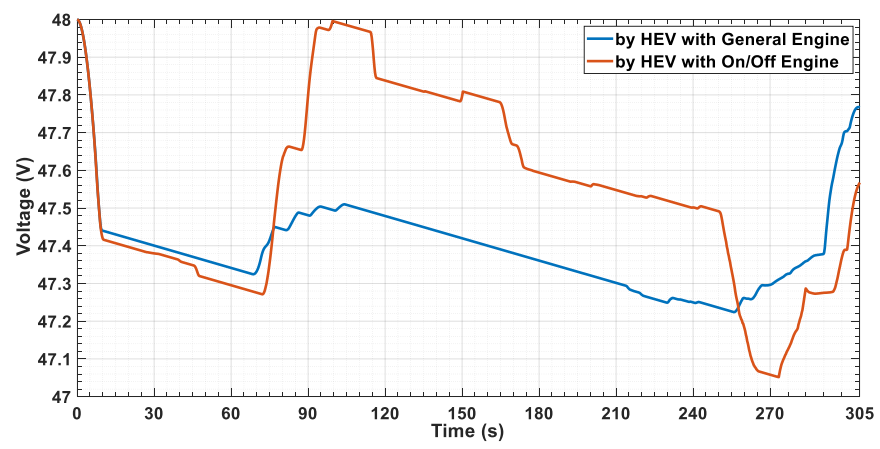

Figure 6. Voltage Trajectories of the HEVs with Two Different Engines

\section{Engine Operation Status}

Fig. 7 illustrates the engine efficiency trajectories of the two configurations along one loop. Note that in both curves, the efficiency of value 0 implies the engine is switched off.
More intuitively, Fig. 8 depicts all the operation points on the engine efficiency map by two online controllers. The white diamonds are from the general engine and the red circles from the on/off engine.

We first compare the engine on/off operations. The general engine is turned on when its rotational speed is larger than the idle speed $(900 \mathrm{rpm})$ and positive torque is demanded by the supervisory controller. Since then, it keeps on until its speed is less than the idle speed. In comparison, the on/off engine is switched on and off 5 times every loop on this testing route.

In Fig. 8, the general engine operation points spread over a larger region on the engine map, but the majority of them are located in the medium and low efficiency sections (0.1 0.28). By contrast, except very few points (less than $3 \%$ of the total), most of the points from the on/off engine are located within or near by the peak efficiency region. Evidently, this engine is of visibly higher efficiency than its counterpart.

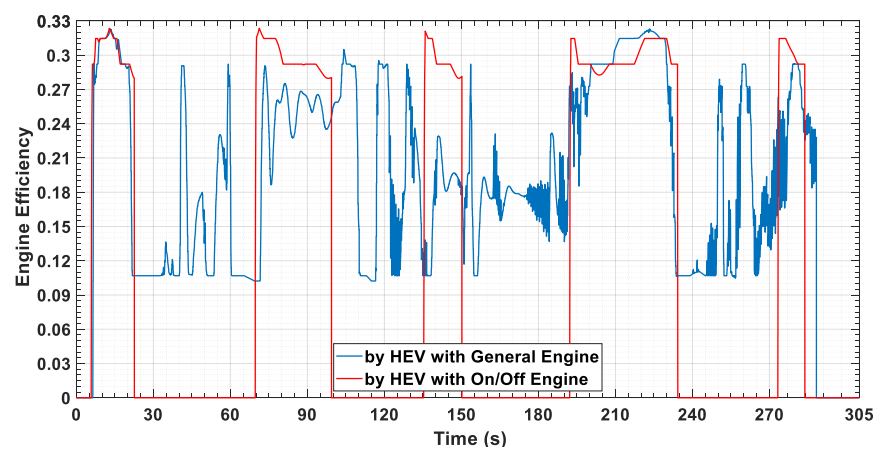

Figure 7. Efficiency Trajectories of the Two Different Engines

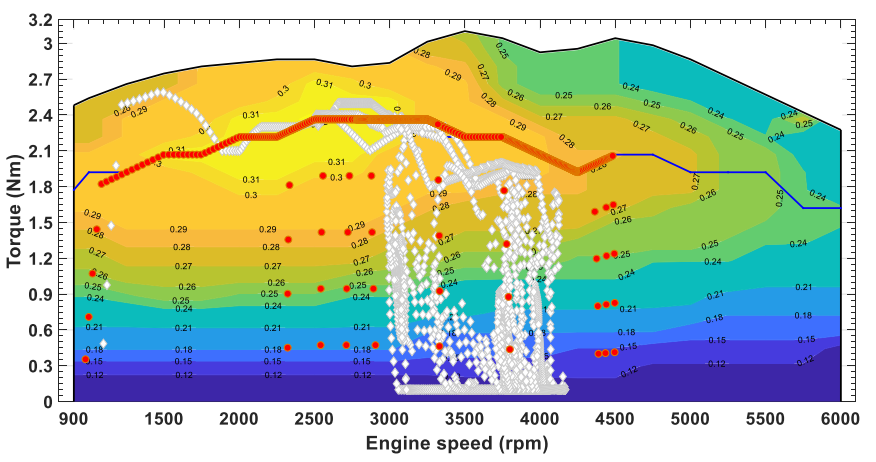

Figure 8. Engine Operation Points by Two Online Controllers

\section{Equivalent Fuel Efficiency}

The results from the two controllers are listed in Table I. For the sake of fairness, the driving time is set as 305 second and the terminal deviation of the voltage is transformed into the equivalent penalty on fuel consumption.

As expected, the HEV with the on/off engine has better fuel efficiency because the engine works with close-to-peak efficiency at most cases; otherwise it is turned off. Despite extra energy paid to the multiple switching on/off, it still has better overall fuel efficiency because this part can be compensated and rewarded by the abundant fuel saved via highly efficient operations. The simulation results indicate that there is no significant difference in distance or terminal voltage deviation, but the special design and the binary control on engine improve the final energy efficiency by $13.07 \%$. 
TABLE I. EQUIVALENT FUEL EFFICIENCY UNDER DIFFERENT CONTROL STRATEGIES

\begin{tabular}{|c|c|c|}
\hline Engine Configuration & General Engine & On/Off Engine \\
\hline Fuel Consumption $(\boldsymbol{m} \boldsymbol{L})$ & 11.698 & 10.193 \\
\hline Voltage Deviation $(\boldsymbol{V})$ & 0.234 & 0.433 \\
\hline Equivalent Cost $(\boldsymbol{m} \boldsymbol{L})$ & 11.848 & 10.469 \\
\hline Distance $(\boldsymbol{k m})$ & 2.265 & 2.263 \\
\hline Efficiency $(\boldsymbol{k m} / \boldsymbol{L})$ & 191.161 & 216.141 \\
\hline
\end{tabular}

\section{CONCLUSION AND FUTURE WORK}

This paper introduces a novel control strategy on the engine of a parallel hybrid electric racing car, so that the engine always works with close-to-peak efficiency or is turned off to avoid unnecessary fuel consumption. The simulation results over an HEV racing route show that, compared to the optimal control of a general engine, this strategy achieves about $13 \%$ increase on equivalent fuel efficiency without violating any system constraints.

Motivated by the potential advantages, we will study the technologies to redesign the engine for larger peak energy efficiency and less emission at the peak efficiency point. The redesign may cause worse efficiency or more emission in other areas of the operation range. But the proposed on/off control strategy can ensure the redesigned engine to always operate within the enhanced region. The redesigned engine and the new control strategy can further increase the energy efficiency and reduce emission of future HEVs.

\section{REFERENCES}

[1] L. Guzella and A. Sciaretta, Vehicle Propulsion Systems: Introduction to Modeling and Optimization, Third Edit., Springer-Verlag, Berlin Heidelberg, 2013.

[2] X. Zhang and C. Mi, Vehicle Power Management: Modeling, Control and Optimization, Springer-Verlag, London, 2011.

[3] V. Wouk, "Hybrid Electric Vehicles," Sci. Am., vol. 277, no. 4, pp. 7074, Oct. 1997.

[4] W. Liu, Introduction to Hybrid Vehicle System Modeling and Control, John Wiley \& Sons, Inc., Hoboken, New Jersey, 2013.

[5] P. Elbert, T. Nuesch, A. Ritter, N. Murgovski, and L. Guzzella, "Engine On/Off control for the energy management of a serial hybrid electric bus via convex optimization," IEEE Trans. Veh. Technol., vol. 63, no. 8, pp. 3549-3559, 2014.

[6] F. R. Salmasi, "Control Strategies for Hybrid Electric Vehicles: Evolution, Classification, Comparison, and Future Trends," IEEE Trans. Veh. Technol., vol. 56, no. 5, pp. 2393-2404, 2007.

[7] Z. Chen and C. C. Mi, "An adaptive online energy management controller for power-split HEV based on dynamic programming and fuzzy logic," 5th IEEE Veh. Power Propuls. Conf. VPPC'09, Dearborn, MI, USA, pp. 335-339, 2009.

[8] S. Onori, L. Serrao, and G. Rizzoni, Hybrid Electric Vehicles:Energy Management Strategies, Springer-Verlag, London, 2016.

[9] Shell Global, "ABOUT SHELL ECO-MARATHON." [Online]. Available: https://www.shell.com/energy-and-innovation/shellecomarathon/about.html. [Accessed: 15-Mar-2019].

[10] Shell Global, "SHELL ECO-MARATHON 2019 OFFICIAL RULES CHAPTER I," 2019. [Online]. Available: https://www.shell.com/makethe-future/shell-ecomarathon/europe/for-europe-participants/_jcr content/par/textimage.stream/1538364518269/90098d1f7999992844e ca9831311f10a1aa95ad5327339dd0765799426bcb1e8/shell-ecomarathon-2019-global-rules-chapter-1.pdf. [Accessed: 15-Mar-2019].

[11] L. Serrao and S. Onori, "Optimal energy management of hybrid electric vehicles including battery aging," $2011 \mathrm{Am}$. Control Conf. ACC, San Francisco, CA, USA, pp. 2125-2130, 2011.

[12] M. Khodabakhshian, L. Feng, and J. Wikander, "One-step prediction for improving gear changing control of HEVs," J. Robot. Mechatronics, vol. 26, no. 6, pp. 799-807, 2014.
[13] N. J. Schouten, M. A. Salman, and N. A. Kheir, "Energy management strategies for parallel hybrid vehicles using fuzzy logic," Control Eng. Pract., vol. 11, no. 2, pp. 171-177, 2003.

[14] J. Peng, H. He, and R. Xiong, "Rule based energy management strategy for a series-parallel plug-in hybrid electric bus optimized by dynamic programming," Appl. Energy, vol. 185, pp. 1633-1643, 2017.

[15] J. P. Trovão, P. G. Pereirinha, H. M. Jorge, and C. H. Antunes, "A multilevel energy management system for multi-source electric vehicles - An integrated rule-based meta-heuristic approach," Appl. Energy, vol. 105, pp. 304-318, 2013.

[16] S. Zhang, R. Xiong, and F. Sun, "Model predictive control for power management in a plug-in hybrid electric vehicle with a hybrid energy storage system," Appl. Energy, vol. 185, pp. 1654-1662, 2017.

[17] C. Sun, X. Hu, S. J. Moura, and F. Sun, "Velocity Predictors for Predictive Energy Management in Hybrid Electric Vehicles," IEEE Trans. Control Syst. Technol., vol. 23, no. 3, pp. 1197-1204, 2015.

[18] L. Serrao, S. Onori, and G. Rizzoni, "ECMS as a realization of Pontryagin's minimum principle for HEV control," $2009 \mathrm{Am}$. Control Conf. ACC, St. Louis, MO, USA, pp. 3964-3969, 2009.

[19] Z. Yuan, L. Teng, S. Fengchun, and H. Peng, "Comparative study of dynamic programming and pontryagin's minimum principle on energy management for a parallel hybrid electric vehicle," Energies, vol. 6, no. 4, pp. 2305-2318, 2013.

[20] M. Khodabakhshian, L. Feng, and J. Wikander, "Improving fuel economy and robustness of an improved ECMS method," IEEE Int. Conf. Control Autom. ICCA, Hangzhou, China, pp. 598-603, 2013.

[21] T. Liu, L. Feng, M. Hellgren, and J. Wikander, "Increasing Fuel Efficiency of a Hybrid Electric Competition Car by a Binary Equivalent Consumption Minimization Strategy," 14th IEEE Int. Conf. Autom. Sci. Eng. CASE, Munich, Germany, pp. 1-7, 2018.

[22] T. Liu, Y. Zou, D. Liu, and F. Sun, "Reinforcement learning-based energy management strategy for a hybrid electric tracked vehicle," Energies, vol. 8, no. 7, pp. 7243-7260, 2015.

[23] Z. Kong, Y. Zou, and T. Liu, "Velocity Predictions Research and Prediction Horizon Determination," Energy Procedia, vol. 105, pp. 3616-3622, 2017.

[24] O. Sundström, L. Guzzella, and P. Soltic, "Optimal Hybridization in Two Parallel Hybrid Electric Vehicles using Dynamic Programming," IFAC Proc. Vol., vol. 41, no. 2, pp. 4642-4647, 2008.

[25] C. Donitz, I. Vasile, C. Onder, and L. Guzzella, "Dynamic programming for hybrid pneumatic vehicles," $2009 \mathrm{Am}$. Control Conf. ACC, St. Louis, MO, USA, pp. 3956-3963, 2009.

[26] Z. Chen, C. C. Mi, B. Xia, and C. You, "Energy management of powersplit plug-in hybrid electric vehicles based on simulated annealing and Pontryagin's minimum principle," J. Power Sources, vol. 272, pp. 160$168,2014$.

[27] R. Wang and S. M. Lukic, "Dynamic programming technique in hybrid electric vehicle optimization," 2012 IEEE Int. Electr. Veh. Conf. IEVC, Greenville, SC, USA, 2012.

[28] B. Bader, O. Torres, J. A. Ortega, G. Lux, and J. L. Romeral, "Predictive real-time energy management strategy for PHEV using lookup-tablebased Dynamic Programming," 2013 World Electr. Veh. Symp. Exhib. EVS27, Barcelona, Spain, 2014.

[29] O. Sundström and L. Guzzella, "A generic dynamic programming Matlab function," 18th IEEE Int. Conf. Control Appl. CCA, St. Petersburg, Russia, pp. 1625-1630, 2009.

[30] O. Sundström, D. Ambühl, and L. Guzzella, "On Implementation of Dynamic Programming for Optimal Control Problems with Final State Constraints," Oil\&Gas Sci. Technol. - Rev.IFP, vol. 65, no. 1, pp. 91$102,2009$.

[31] K. J. Åström and R. M. Murray, Feedback systems: An Introduction for Scientists and Engineers, First Edit., Princeton University Press, Princeton, New Jersey, 2008.

[32] K. J. Åström and B. Wittenmark, Computer-Controlled Systems: Theory and Design, Third Edit. Dover Publications, Inc., Mineola, New York, 2011.

[33] Technische Hochschule Mittelhessen, "SHELL ECO-MARATHON EUROPE 2016:LONDON TRACK MAP." [Online]. Available: https://www.thm.de/m/images/Labore/LfMS/Strecken/sem-europe2016-london-track-map.pdf. [Accessed: 15-Mar-2019].

[34] H. M. Sweeney, "The Design of a Driventrain for an Eco-Marathon Vehicle," 2016. [Online]. Available: https://www.slideshare.net/ HughMcSweeney/reportdraft2. [Accessed: 15-Mar-2019]. 